\title{
Extracting Method of Projectile Spin Speed based on Hilbert-Huang Transform
}

\author{
Duan Peng-wei ${ }^{1, a^{*}}$, Liu Zhi-xue ${ }^{1, b}$, Gong Zhi-hua ${ }^{1, c}$, \\ Leng Xue-bing ${ }^{1, d}$ and Dong Li-tao ${ }^{1, e}$ \\ ${ }^{1}$ Baicheng Ordnance Test Center of China, Baicheng 137001, Jilin \\ adpw68909@163.com, bliuzx09@qq.com, cgzh63298@126.com, \\ dxb200508@sina.com, e448713843@qq.com
}

Keywords: micro Doppler effect; CW radar; HHT; spin speed; FFT.

\begin{abstract}
Making use of micro Doppler Effect measured by continuous wave radar (CW radar), the micro Doppler modulation mathematical model of radar echo cased by projectile rotation is established, and the analytic relationship between projectile rotation and micro motion is deduced. Based on Hilbert-Huang Transform (HHT), a new method of extracting spin speed is proposed to instead the traditional FFT method. Through the simulation, the new method is verified to be effective. This method is applied to extracting spin speed form the measured data, and its result is compared to the result calculated by FFT method. The result shows that the new method could effectively extract spin speed, and accurately describe the change of spin speed along with time.
\end{abstract}

\section{Introduction}

The spin speed is an important test parameter of motive projectile. It is directly related to the design of various projectiles, guidance devices and fuses etc. It is also a key parameter for flying stability, ballistic trajectory and impact point accuracy in the test and use of the projectile. There are mainly telemetry method and optical method to measure projectile spin speed in early stage, and all of these methods have some defects [1]. Some sensors must be added upon the projectile with the telemetry method, so it will change the structure of projectile. To the optical method, it is affected greatly by the environment, and it could only measure a little part of the ballistic trajectory. In recent years, through grooving the bottom of the projectile, spin speed is measured by continuous wave Doppler radar. Compared to early methods, this method can measure the spin speed of projectile along with time, but it also changes the structure of projectile. On the basis of that method, reference [2] proposed a spin speed measuring method based on the micro motion. This method needs not change structure of projectile, and it can operate for a long range, but it still uses traditional FFT to calculate spin speed. FFT is restricted by the exclusion principle of frequency resolution, so it is a low resolution method. Therefore, a new method based on HHT is proposed to extract spin speed by processing the radial velocity measured by $\mathrm{CW}$ radar.

\section{Modulation Model of Projectile Rotation}

In the coordinate system of projectile axis [3], a scattering point $P$ is $l$ away from the center of the projectile bottom shown as Fig. 1. So its coordinate is shown as Eq.1.

$$
\left[\begin{array}{l}
x(t) \\
y(t) \\
z(t)
\end{array}\right]=\left[\begin{array}{l}
0 \\
l \sin (\omega t) \\
l \cos (\omega t)
\end{array}\right]
$$




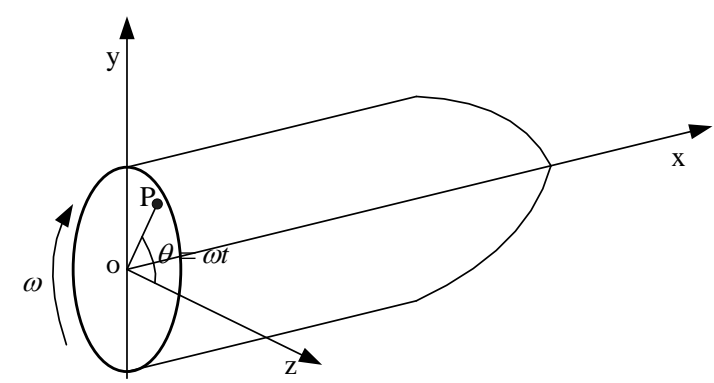

Fig. 1. Rotation diagram of projectile.

Radar measurement coordinate system $O X Y Z$ and reference coordinate system $o X_{N} Y_{N} Z_{N}$ is established shown as Fig. 2, and the $\mathrm{X}$ axis of the Radar measurement coordinate system parallels the firing azimuth of the gun. The origin of reference coordinate is the center of the projectile bottom, and the axes of reference coordinate system parallel the axes of Radar measurement coordinate system. Reference coordinate system moves with the projectile bottom.

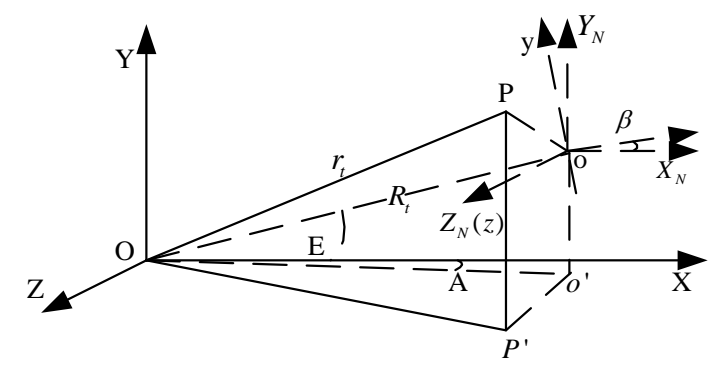

Fig. 2. Relationship of three coordinate systems.

After twice rotations, reference coordinate system can be transformed into the coordinate system of projectile axis. First, reference coordinate system rotates around the $\mathrm{Z}$ axis with $\beta$, and then rotates around the new $\mathrm{Y}$ axis with $\psi$. $\beta$ is elevation of the projectile axis and $\psi$ is azimuth of the projectile axis. When the projectile flies in the air, azimuth of the projectile axis $\psi$ is usually very small, so it can be considered $\psi=0$ here. Then the coordinate of $P$ in the reference coordinate system is shown as Eq.2.

$$
\left[\begin{array}{l}
X_{N}(t) \\
Y_{N}(t) \\
Z_{N}(t)
\end{array}\right]=\left[\begin{array}{ccc}
\cos \beta & -\sin \beta & 0 \\
\sin \beta & \cos \beta & 0 \\
0 & 0 & 1
\end{array}\right]\left[\begin{array}{l}
0 \\
l \sin (\omega t) \\
l \cos (\omega t)
\end{array}\right]=\left[\begin{array}{c}
-l \sin \beta \sin (\omega t) \\
l \cos \beta \sin (\omega t) \\
l \cos (\omega t)
\end{array}\right]
$$

Usually, radar is placed close to the firing azimuth of the gun, so the measuring azimuth $A$ of radar is very small, and so it also can be considered $A=0$ here. Then the slant range $r_{t}$ between $\mathrm{P}$ and radar is shown as Eq.3.

$$
r_{t}=\sqrt{O Q^{2}+Q S^{2}+S P^{2}}=\sqrt{\left(R_{t} \cos E+X_{N}(t)\right)^{2}+\left(R_{t} \sin E+Y_{N}(t)\right)^{2}+\left(Z_{N}(t)\right)^{2}}
$$

Because $R_{t}>>X_{N}(t), Y_{N}(t), Z_{N}(t), r_{t}$ can be approximated as Eq.4.

$$
r_{t} \approx R_{t}+X_{N}(t) \cos E+Y_{N}(t) \sin E
$$

Substitute Eq.2 into Eq.4, then

$$
r_{t}=R_{t}-l \sin \beta \sin (\omega t) \cos E+l \cos \beta \sin (\omega t) \sin E=R_{t}+l \sin (E-\beta) \sin (\omega t)
$$


In a short time, $E$ and $\beta$ change little, so they can be considered as constant. If $a=l \sin (E-\beta)$, then

$$
r_{t}=R_{t}+a \sin (\omega t)
$$

Therefore, the radial velocity of $P$ to radar is shown as Eq.7

$$
v_{r p}(t)=\dot{R}_{t}+a \omega \cos (\omega t)=\dot{R}_{t}+b \cos (\omega t)=v_{r o}+v_{r m d}
$$

In Eq.7, $v_{r o}=\dot{R}_{t}$ is the radial velocity of the projectile to radar. $v_{r m d}=b \cos (\omega t)$ is velocity of micro motion cased by rotation of projectile, and it follows the sinusoidal variation. $b=a \omega$ is amplitude of micro motion velocity. So the radial velocity of the projectile is the sum of the radial translation velocity and the micro motion velocity. The change frequency of micro motion velocity is the spin speed of projectile.

\section{Extracting method of projectile spin speed based on HHT}

On the basis of above analysis, instantaneous phase of the rotating projectile can be calculated from the radial velocity of projectile measured by radar with HHT, and the spin speed can be extracted by phase difference. According to the HHT, there are two steps to calculate spin speed. First step is empirical mode decomposition (EMD), and another is instantaneous phase difference to calculate spin speed.

EMD. EMD is a process to sift the signals that decomposes a complex signal into some intrinsic mode function (IMF) signals. These signals have physical significance, and each of them has only one frequency component in every moment.

IMF has two conditions:

1) In the whole signal, the numbers of zero-crossing points and local extreme points must be equal or different at only one.

2) At any point, the mean values of the envelope determined by local maximum and the envelope determined by the local minimum are zero.

According to these two conditions, the radial velocity of the projectile is decomposed into IMF in the EMD way [4] shown as Eq.8.

$$
X(t)=\sum_{i=1}^{n} C_{i}(t)+R_{m}(t)
$$

In Eq.8, $C_{i}(t)$ is IMF signal, and $R_{m}(t)$ is the residual function, indicating the overall trend of the signal. Spin speed calculation. Transforming the IMF signal of micro motion velocity with Hilbert transform, analytic phase of micro motion velocity is calculated. Then the spin speed of micro motion velocity can be determined from the derivative of signal phase. $v_{r m d}$ can be transform into $v_{r m d H}$ :

$$
v_{r m d H}(t)=\frac{1}{\pi} p \cdot v \cdot \int \frac{v_{r m d}(t)}{t-\tau} d \tau
$$

In Eq.9, p.v is the Cauchy principal value integrals. Form the nature of the Hilbert transform, the analytic function of the real signal can be got as followed.

$$
S(t)=v_{r m d}(t)+j v_{r m d H}(t)=a(t) e^{j \theta(t)}
$$

In Eq.10, $a(t)=\sqrt{v_{r m d}^{2}(t)+v_{r m d H}^{2}(t)}$ is the instantaneous amplitude, $\theta(t)=\arctan \left[\frac{v_{r m d}(t)}{v_{r m d H}(t)}\right]$ is the instantaneous phase.

The spin speed which is also instantaneous frequency of $v_{r m d}$ defined as Eq.11. 


$$
f(t)=\frac{1}{2 \pi} \omega(t)=\frac{1}{2 \pi} \frac{d \theta(t)}{d t}
$$

\section{Simulation computation}

Translational velocity curve of projectile can be approximated by the high order polynomial, so it is assumed that radial velocity of projectile centroid is parabolic curve. The simulation conditions are listed as follow.

The radial translation velocity of the projectile is $v_{d}=0.2 t^{2}+15 t+660 \mathrm{~m} / \mathrm{s}$. The spin speed of the projectile is $f_{r}=(150-4 t) \mathrm{Hz}$. The micro motion velocity of the projectile is $v_{r}=0.1 \cos (2 \pi(150-2 t) t)$. The velocity measuring random error is $0.02 \mathrm{~m} / \mathrm{s}$.

Then radial velocity of the projectile measured by radar can be expressed as Eq.12.

$$
v=0.2 t^{2}+15 t+660+0.1 \cos (2 \pi(150-2 t) t)+0.02 N(t)
$$

In Eq.12, $N(t)$ is random number that obeys the $(0,1)$ normal distribution.

According the above steps, the simulated radial velocity of the projectile is processed.

First, the radial velocity is mirrored extended [5] at both ends. Through EMD, radial velocity is decomposed to radial translation velocity and micro motion velocity that shown as Fig. 3.
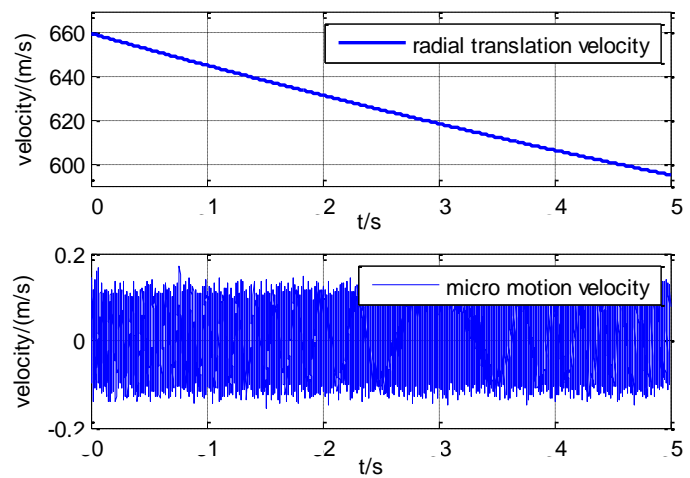

Fig. 3. Radial translation velocity and micro motion velocity decomposed from radial velocity.

Then, instantaneous phase can be calculated from micro motion velocity by Hilbert Transform. After unwrapping and fitting of the instantaneous phase, the spin speed is extracted by Phase difference shown as Fig. 4.
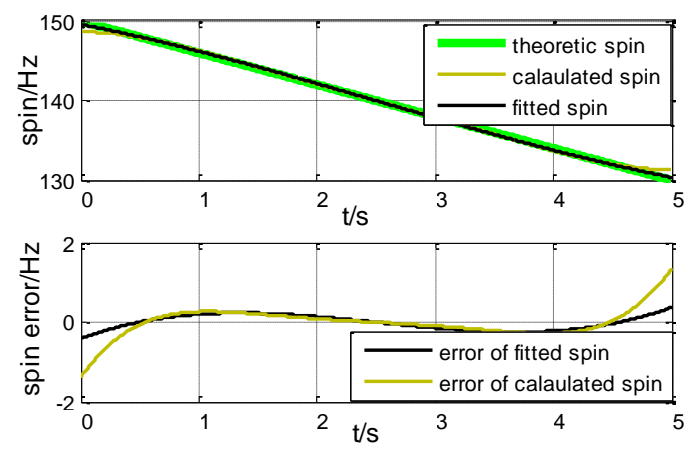

Fig. 4. Comparison of calculated spin speed and theoretical spin speed.

It is shown from Fig. 4 that, even though the radial velocity is mirror extended, there is still end effect in calculated spin speed. The errors are about $1.4 \mathrm{~Hz}$ at the two ends where the errors are the maximum. Fitted spin speed can be got by deleting part data at the ends and fitting the calculated spin speed. The error of fitted spin speed is less than $0.5 \mathrm{~Hz}$. 


\section{Application}

Selecting radial velocity (40s 45s) of a projectile measured by a CW radar as original data, the spin speed of projectile is calculated with method proposed in the paper and the traditional FFT method. The results are shown as Fig. 5.

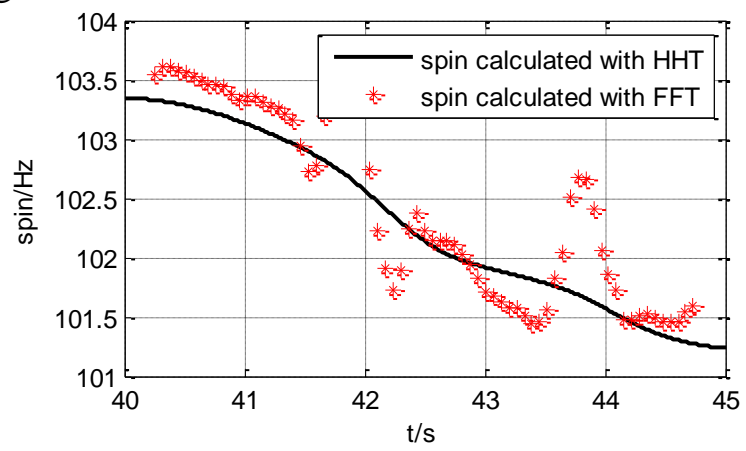

Fig. 5. Comparison of spin speed calculated with HHT and FFT.

Through comparison, FFT method is restricted by the exclusion principle of frequency resolution. If more FFT point is selected, there is not only one peak in the spectrum, and it cannot determine the frequency. If the number of FFT point is fewer, the frequency resolution will be reduced. Even after compensating and correcting, the frequency resolution still cannot meet the need of calculation. So FFT method is low precision method. The method proposed in the paper can effectively extract the spin speed of projectile from radial velocity. Its results are smoother and its error is smaller. Besides, it does not decrease data rate, and it describes spin speed changed with time more accurate.

\section{Conclusion}

The modulation model of the projectile rotation is established based on micro motion and the relationship between radial velocity of projectile measured by $\mathrm{CW}$ radar and micro velocity caused by projectile rotation is obtained. It is deduced that the spin speed of projectile and the change frequency of micro velocity are equal. Form the nature and advantage of the HHT, it is proposed to process the radial velocity with HHT. Radial velocity is decomposed into translation velocity and micro velocity by EMD, and then derivative of instantaneous phase of micro velocity is spin speed of projectile. From simulation, it is concluded that the method proposed in the paper can effectively extract the spin speed of projectile from radial velocity. From application and comparison with FFT method, it is concluded that the new method are smoother and its error is smaller. Besides, it does not decrease data rate, and it describes spin speed changed with time more accurate.

\section{References}

[1] Jin Da-gen, Ren Guo-min. Experimental exterior ballistic theory. The Publishing House of Ordnance Industry, Beijing, 1991: 31-134.

[2] Zhang Wan-jun, Wu Xiao-ying, Leng Xue-bing, etc. Testing method of projectile rotating speed based on micro-Doppler effect of CW radars. Journal of Academy of Armored Force Engineering, 2012, 26(5): 47-50.

[3] Tao Ru-yi, Wang Hao, Huang Bei. The model and simulation of aerial dispersing on piston dispersal mechanism of cluster munition. ACTA ARMAMENTARII, 2009, 30(3): 282-288.

[4] Song Shi-de, Yao Zhi-chao, Wang Xiao-na. The summary of Hilbert-Huang transform. The International Society for Optical Engineering, 2013, Vol. 8907, 890734.

[5] Ju Ji-tao, Dai Ben-qi, Wu Hao, etc. A time-frequency analysis method for non-stationary signals based on Hilbert-Huang Transform and its application. Micricomputer Information, 2009, 25(8-1): 154-156. 